\title{
Editorial
}

\section{La Ingeniería de Control y su Rol Actual en la Medicina}

\author{
Jose F. Garcia-Tirado, Ph.D \\ Instituto Tecnológico Metropolitano, Facultad de Ciencias Económicas y Administrativas, Grupo de Investigación en Calidad, \\ Metrología y Producción, Calle 73 No. 76A - 354 Vía al Volador, 050034, Medellín - Colombia
}

En la actualidad, a pesar del gran avance tecnológico al que continuamente estamos expuestos, todavía existe un gran desconocimiento acerca de la importancia y participación de la ingeniería de control en todas las ramas de la industria y de la tecnología moderna. En este sentido, es aún más desconocido el rol que tiene esta ingeniería en una gran cantidad de procedimientos clínicos y en general como sustento teórico para dar explicación a la gran cantidad de procesos fisiológicos que posee el cuerpo humano.

La ingeniería de control es una disciplina de la ingeniería que hace uso de la teoría de control con el objetivo de diseñar sistemas (controladores) que garanticen un comportamiento deseado de otros sistemas (planta). La teoría de control, a su vez, es una rama interdisciplinaria de la ingeniería y las matemáticas que estudia principalmente el comportamiento de los sistemas dinámicos y la manera de lograr que estos se comporten de manera deseada. El término "sistema" en este contexto se usa como una abstracción del mundo real que es susceptible de recibir excitaciones (entradas) y que ante estas entradas exhibe unas respuestas (salidas).

Uno de los conceptos más usados en ingeniería de control es el concepto de retroalimentación. La retroalimentación es un principio mediante el cual la información proveniente de un sensor (salida de la planta) es contrastada con respecto a una consigna de control (referencia) con el ánimo de darle orden a un actuador (variable manipulada) para ejecutar una acción correctiva. Un ejemplo intuitivo de sistema de control en lazo cerrado o control por retroalimentación es el control de la velocidad de crucero en un automóvil: después de ajustar la velocidad deseada, por ejemplo $60 \mathrm{~km} / \mathrm{h}$, el controlador recibe la medición hecha por el sensor de velocidad. Una vez se detecta alguna desviación de la consigna de control (velocidad deseada), el controlador calcula la acción correctiva y la envía al acelerador (actuador) hasta que se cumpla la consigna deseada.

En el contexto biomédico, el concepto de "sistema de control" ha tenido un auge significativo en los últimos años, aunque los primeros registros de la unión de estas dos disciplinas datan de finales del siglo antepasado con el trabajo de Claude Bernard "Millieu Intérieur" el cuál ya resaltaba el comportamiento regulatorio de varios sistemas fisiológicos del cuerpo humano en respuesta a perturbaciones externas o ambientales [1]. En este sentido, "la planta" ya no es un equipo industrial, una planta de procesos, un sistema eléctrico, etc., sino que es un ser vivo con múltiples controladores automáticos internos haciendo su trabajo de manera colaborativa y distribuida. Algunos ejemplos de tales reguladores naturales, en el caso puntual del ser humano, son los mecanismos homeostáticos de temperatura, $\mathrm{pH}$ de fluidos extracelulares, iones de calcio y sodio, glucosa en sangre, entre otros. Estos controladores naturales son tan complejos que aún la ingeniería no ha llegado a tales niveles de detalle a pesar de todos los esfuerzos que se han hecho y de tantos avances que se han logrado.

A pesar de la alta complejidad de los sistemas biológicos, debe resaltarse que el modelado matemático ha ayudado ostensiblemente a la comprensión de diversos fenómenos fisiológicos y que esta comprensión detallada ha dado lugar a diferentes metodologías de tratamiento de diversas patologías, incluyendo metodologías automatizadas. Entre las aplicaciones más destacadas y exitosas de sistemas de control en biomedicina se encuentran principalmente los relacionados con el sistema cardiovascular y con el sistema endocrino [2]. Sin embargo, también se han presentado avances significativos en control de presión arterial, infusión automática de anestesia y en el control automático de la enfermedad vascular periférica.

Con respecto al sistema cardiovascular, existe un conocimiento popular más cercano gracias al dispositivo denominado marcapasos. Este dispositivo es una bomba mecánica que envía pulsos eléctricos de baja intensidad al corazón para ayudarlo a contraerse y así controlar la frecuencia cardíaca principalmente en pacientes con arritmias. Sin embargo, actualmente siguen existiendo dificultades para emular por completo el comportamiento dinámico del corazón, debido principalmente a la gran cantidad de regímenes de operación a los cuales está expuesto el paciente: nivel de ejercicio, manejo de emociones, posturas, etc. Según Doyle, los avances más recientes en esta materia incluyen análisis dinámico en tiempo real, controladores adaptables y controladores predictivos capaces de compensar cambios según las necesidades del paciente [2].

En el caso del sistema endocrino, quizá el área más activa actualmente en términos de investigación en relación con la ingeniería de control, es la relacionada con el desarrollo de un páncreas artificial. Este dispositivo es un sistema artificial de control que surge como tratamiento alternativo a la enfermedad Diabetes Mellitus tipo 1 (T1DM, por sus siglas en inglés). En general, se conoce como Diabetes Mellitus (DM) a la incapacidad del páncreas de producir la hormona insulina o al deterioro 
metabólico que surge cuando el cuerpo humano no puede usar esta hormona de manera adecuada. En el caso puntual de la T1DM, el páncreas no produce insulina debido a un ataque autoinmune que destruye las células beta productoras de esta hormona. Dado que las células beta son finitas, su destrucción conlleva a la deficiencia completa de la producción de esta hormona.

La insulina juega un papel muy importante porque transporta la glucosa para que sea ingresada a diversas células del cuerpo humano. Sin insulina, la glucosa no puede ser captada por las células y la concentración de ésta molécula en el cuerpo humano se eleva, lo que comúnmente se conoce como hiperglicemia, la cual es una condición que a largo plazo es mortal. Con la ruptura del lazo de control natural, el cuerpo humano queda en un estado de "lazo abierto", quedando en un estado de vulnerabilidad permanente hacia condiciones de alta concentración de glucosa (hiperglicemia) y baja concentración de glucosa (hipoglucemia). La hipoglucemia o estado de baja concentración de glucosa, a diferencia de la hiperglicemia, puede ser mortal a corto plazo si no se trata de manera adecuada.

Puede decirse que la génesis del páncreas artificial fue gracias al trabajo seminal de Bolie en 1961 [3]. En este trabajo, se propuso un primer modelo matemático para caracterizar el comportamiento dinámico del sistema glucosa-insulina durante una prueba intravenosa de tolerancia a la glucosa (IVGTT, por sus siglas en inglés). A partir de este trabajo se comenzó una línea de investigación de caracterización dinámica de animales y seres humanos ante diferentes tipos de test de tolerancia a la glucosa y a la insulina entre los cuales se resaltan los realizados por Bergman, Cobelli y Colaboradores a partir del famoso Modelo Mínimo de Bergman [4].

A partir del modelo mínimo de Bergman, se han hecho diferentes intervenciones en aras de emular el comportamiento dinámico del mecanismo homeostático de la glucosa del ser humano en su diario vivir. Actualmente, este modelo matemático evolucionó a lo que se conoce actualmente como modelo UVa/Padova, nombre derivado de la sinergia entre las Universidades de Virginia en EEUU y de Padova en Italia [5]. Este modelo matemático cuenta actualmente con la aprobación de la Administración de Medicamentos y Alimentos de EEUU (FDA, por sus siglas en inglés) para evitar ensayos preclínicos en animales y así saltar directamente a ensayos clínicos en seres humanos. A pesar de la gran popularidad que cuenta este modelo matemático, existen otras alternativas altamente usadas y citadas como son el modelo de Cambridge y el modelo de Sorensen [6], [7]. Una revisión de los modelos matemáticos más relevantes en el estudio de la T1DM puede encontrarse en [8].

Un modelo matemático con la capacidad de emular adecuadamente tal mecanismo homeostático tendrá la facultad de "orientar" a un controlador basado en este modelo para que tome decisiones de control (infusión de insulina y glucagón) que garanticen la normoglicemia en el paciente. Actualmente, la investigación apunta al desarrollo de modelos más fisiológicos que describan de manera más adecuada el mecanismo homeostático de la glucosa y al desarrollo de mecanismos de identificación paramétrica en línea para ajustar al modelo matemático usado a diferentes estados fisiológicos del paciente.

Actualmente, el proyecto páncreas artificial viene siendo desarrollado por diferentes consorcios de universidades de manera separada. De manera casi obvia, cada consorcio tiene un "know how" que involucra diferentes tecnologías, conocimientos, modelos matemáticos, estrategias de control, así como también diferentes presupuestos de investigación. Lo que es claro aquí, es que la ingeniería de control no existe sola sino como un complemento de un área específica. De aquí el éxito de la carrera aeroespacial, armamentista y vehicular, sólo por mencionar unos ejemplos. En el caso biomédico, es necesario entender que los ingenieros de control somos expertos en diseñar y analizar sistemas complejos, pero prácticamente tenemos muy poco contacto con los médicos. Del mismo modo los médicos no interiorizan las estrategias de control que están (o pueden estarlo de manera artificial) en las patologías que ellos tratan. Por lo tanto, debe hacerse una reflexión en el sentido de conformar paneles interdisciplinarios de ingenieros y médicos para lograr avances más significativos en la medicina.

En el caso colombiano, existe una unión de investigación entre el Instituto Tecnológico Metropolitano, la Universidad Nacional de Colombia - Sede Medellín, el Hospital Pablo Tobón Uribe y la Universidad de Antioquia con el objetivo de desarrollar tecnologías que ayuden un desarrollo de páncreas artificial. Actualmente, se está trabajando en el desarrollo de un modelo semifísico de base fenomenológica del mecanismo homeostático de la glucosa. Este modelo es respetuoso de los primeros principios o balances de materia que se dan entre los órganos participantes de la homeostasis. Sin embargo, este modelo también es práctico y pretende identificar parámetros intra-paciente usando datos reales. Con este modelo matemático se espera contribuir de manera significativa al posterior desarrollo de controladores avanzados que ayuden al tratamiento automatizado de la T1DM.

\section{Referencias}

[1] F. L. Holmes, "Claude Bernard, The 'Milieu Intérieur', and Regulatory Physiology," Hist. Philos. Life Sci., vol. 8, no. 1, pp. 325, 1986.

[2] T. Samad and A. M. Annaswamy, Eds., The Impact of Control Technology. IEEE, 2011.

[3] W. V Bolie, "Coefficients of normal blood glucose regulation," J Appl Physiol., vol. 16, no. 5, pp. 783-788, 1961.

[4] R. N. Bergman, R. N. Bergman, Y. Z. Ider, Y. Z. Ider, C. R. Bowden, C. R. Bowden, C. Cobelli, and C. Cobelli, "Quantitative estimation of insulin sensitivity.," Am. J. Physiol., vol. 236, no. 6, pp. E667-77, 1979. 
[5] C. Dalla Man, F. Micheletto, D. Lv, M. Breton, B. Kovatchev, and C. Cobelli, "The UVA/PADOVA Type 1 Diabetes Simulator: New Features.," J. Diabetes Sci. Technol., vol. 8, pp. 26-34, 2014.

[6] M. E. Wilinska, M. Bodenlenz, L. J. Chassin, H. C. Schaller, L. A. Schaupp, T. R. Pieber, and R. Hovorka, "Interstitial glucose kinetics in subjects with type 1 diabetes under physiologic conditions," Metabolism., vol. 53, no. 11, pp. 1484-1491, 2004.
[7] J. T. Sorensen, "A Phisiologic Model of Glucose Metabolism in Man and its use to Design and Assess Improved Insulin Therapies for Diabetes," Massachusetts Institute of Technology, 1985.

[8] L. Lema-Perez, E. Aguirre-Zapata, and J. Garcia-Tirado, "Recent advances in mathematical models for the understanding and treatment of Type 1 Diabetes Mellitus," in Automatic Control (CCAC), 2015 IEEE 2nd Colombian Conference on, 2015, pp. 1- 